\title{
DWS Model Update and Analysis Using WaterGEMS
}

Caitlynn Hanna, University of Alaska Fairbanks - SIST Program

Jonathan Hunt, Fermilab

\section{Domestic Water Service System (DWS) and the Existing WaterGEMs Model}

The DWS is used to supply water for personal, household, or sanitary purposes. It is of extreme importance to everyone on Fermilab property.

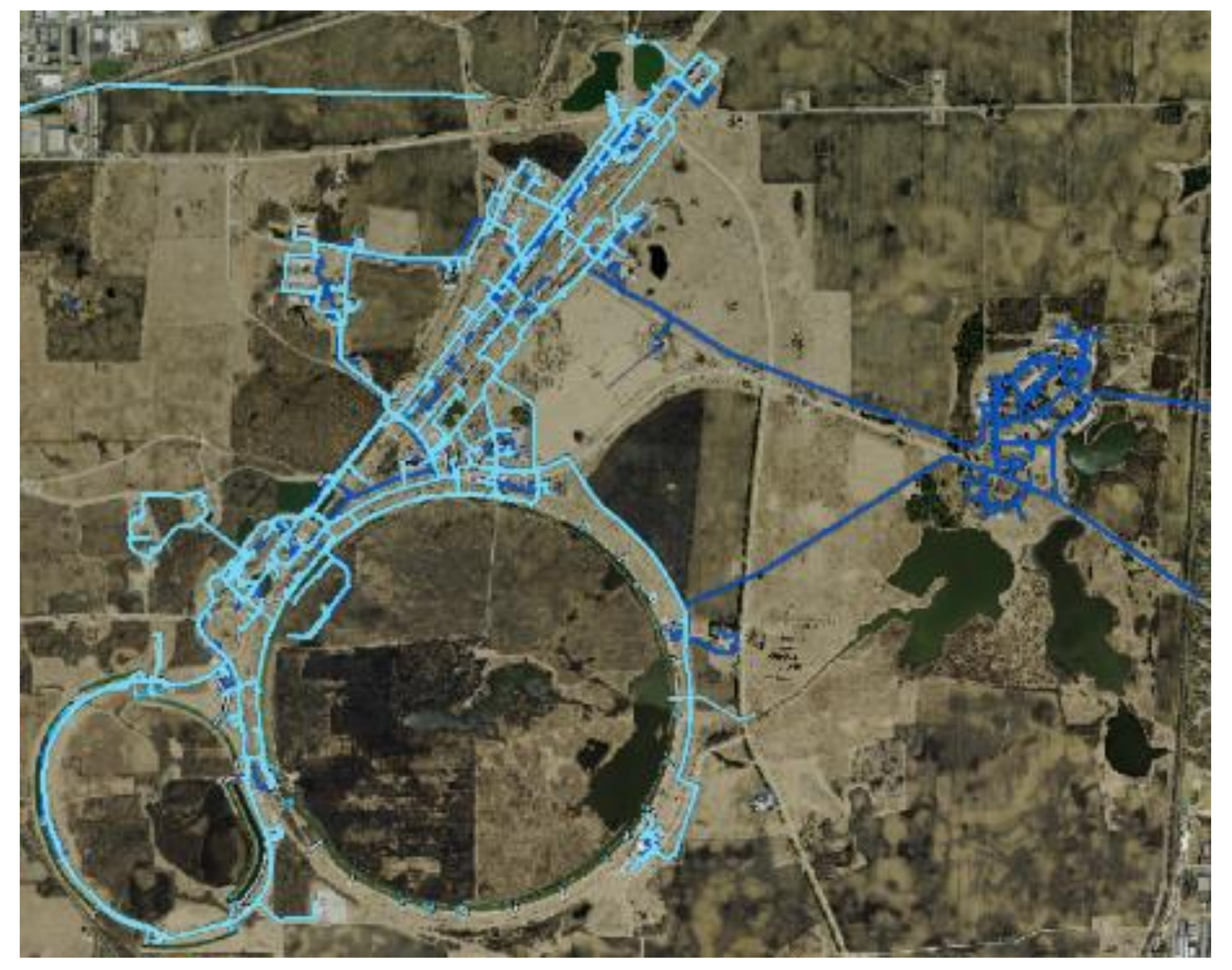

Figure 1: Fermilab GIS, light blue indicates ICW and blue indicates DWS

Fermilab previously has hired consultants to update the DWS and ICW models. In 2019 a SIST intern worked on the ICW model, this summer I was tasked with the DWS model. WaterGEMs is a hydraulic modeling software used to analyze, design, and optimize water distribution systems. The tools utilized were the steady-state analysis, automated fire flow simulations, scenario management, and water quality analysis. Due to the nature and use of domestic water, the system is in compliance with the NEPA and EPA.

\section{Acknowledgement}

This manuscript has been authored by Fermi Research Alliance, LLC under Contract No. DE-AC02-07CH11359 with the U.S. Department of Energy, Office of Science, Office of High Energy Physics.

\section{Understanding and Updating the Model}

The projects to be updated and examined for discrepancies within the DWS model was PIP-II and IERC. PIP-II is still under construction, but the plans were used to update the model to reflect the entirety of the future of PIP-II.
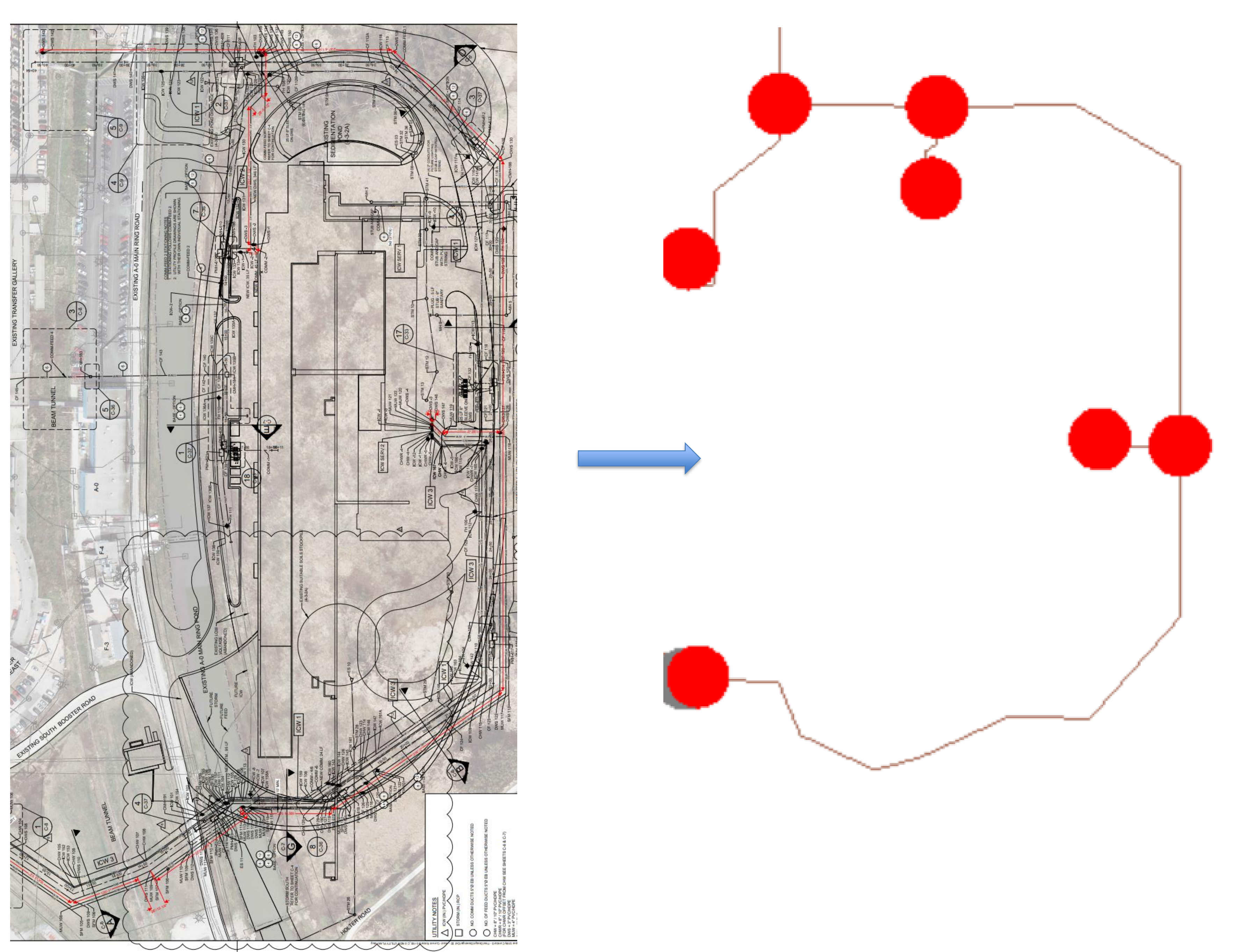

Figure 2: Construction for PIP II and the representation within the model

Data was taken and used to update the model. Lengths, pipe diameter, pipe material, pipe lengths, elevation, and water demand was used to update and ensure the most accurate representation of what was constructed.

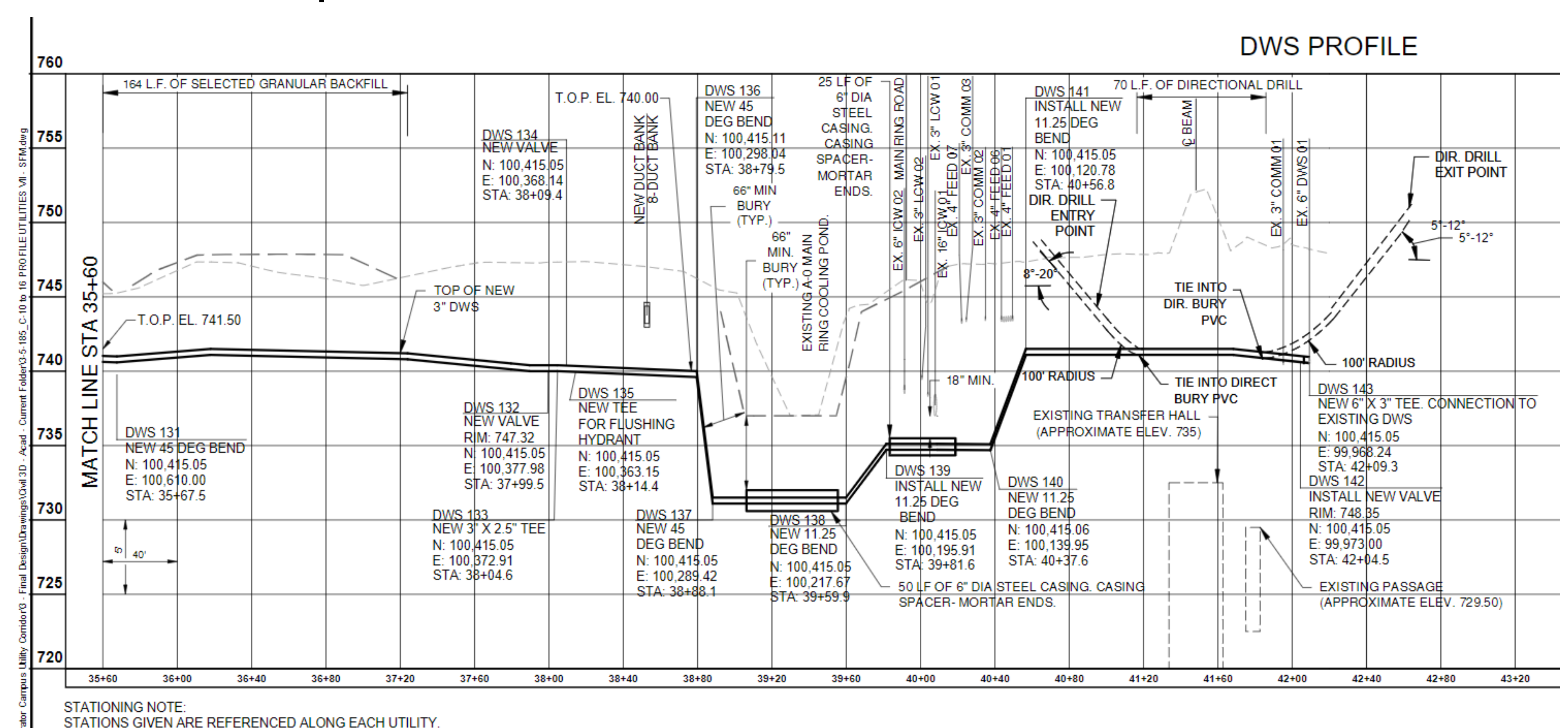

Figure 3: Profile of a section of PIP-II's DWS

\section{Conclusions and Future Projects}

The updated model shows that, with proper management and operation setpoint changes, the existing DWS can handle the current and near future demands at Fermilab. The model is an important tool for understanding the behavior of the DWS. Having an accurate representation of the domestic water service, with factors as close to reality as possible is important for many factors of safety, such as healthy drinking water and fire safety measures.

The new DWS WaterGEMS model summarizes the viability of adding water flow rate to the current system and allows for an efficient initial analysis on the effects of upcoming projects' added demands of the DWS.

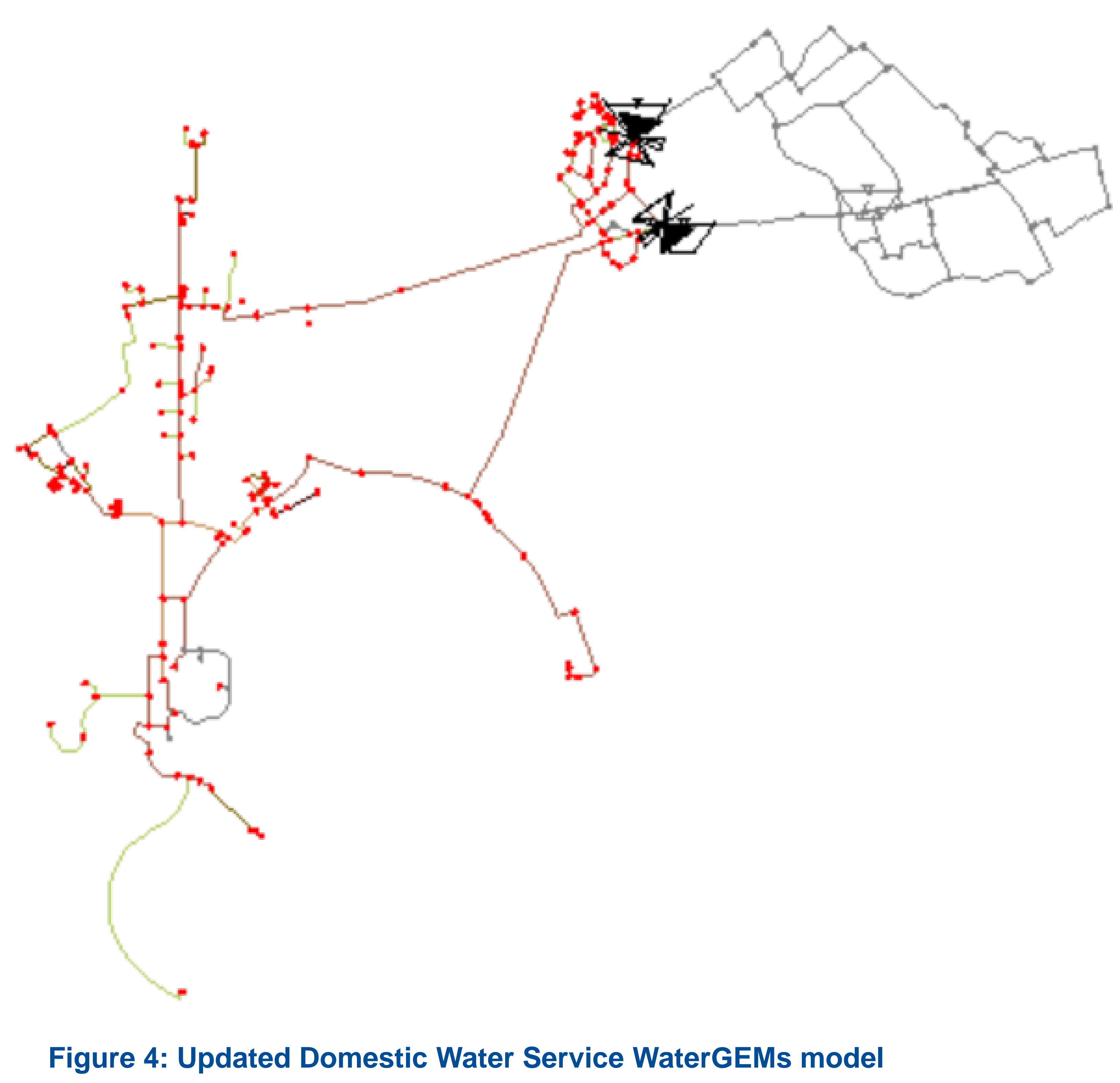

